\title{
A INFLUÊNCIA DO TREINAMENTO NA DISCINESIA ESCAPULAR EM JOGADORAS DE VOLEIBOL: UM ESTUDO PROSPECTIVO
}

\author{
THE INFLUENCE OF TRAINING IN SCAPULAR DYSKINESIA OF VOLLEYBALL PLAYERS: \\ A PROSPECTIVE STUDY
LA INFLUENCIA DEL ENTRENAMIENTO EN LA DISQUINESIA ESCAPULAR EN JUGADORAS
DE VOLEIBOL: UN ESTUDIO PROSPECTIVO

\begin{abstract}
Renato Rozenblit Soliaman (Fisioterapeuta)

Fabrício Lisboa Azzolini'

(Fisioterapeuta)

Ligia Leme' (Fisioterapeuta)

Benno Ejnisman' (Médico)

Alberto de Castro Pochini' (Médico)

Ronaldo Alves da Cunha'

(Fisioterapeuta)

1. Universidade Federal de São Paulo (UNIFESP), Centro de Traumato-Ortopedia do Esporte (CETE), São Paulo-SP, Brasil.
\end{abstract}

\section{Correspondência:}

Rua: Estado de Israel, 636,

Vila Clementino, São Paulo, Brasil.

CEP: 04022-001.

renatorsoliaman@uol.com.br

\section{RESUMO}

Introdução: No movimento de ataque, o equilíbrio do ritmo escapulotorácico exerce papel fundamental na eficiência do gesto esportivo, sendo a discinesia escapular comumente associadas a lesões específicas do ombro do arremessador. Dentre suas causas é frequentemente relatada a fadiga muscular consequente ao uso excessivo da musculatura periescapular. Objetivo: Avaliar a influência do treinamento na presença de discinesia escapular em atletas de voleibol. Métodos: Trata-se de um estudo de coorte prospectivo, no qual foram avaliadas 12 atletas do gênero feminino praticantes de voleibol, que não possuíam cirurgias prévias nos ombros e praticavam no mínimo 10 horas de treinos semanais. Foi realizada avaliação pré e pós treino, no qual realizavam 30 min de aquecimento e 120 min de treino específico da modalidade. A discinesia escapular foi avaliada em 'SIM' ou 'NÃO', definida de acordo com a presença ou ausência de alterações no padrão de movimento escapulotorácico, respectivamente. Resultados: A prevalência de discinesia nesta população foi identificada em nove das doze atletas na avaliação pré-treino (75\%) e após a realização do treinamento uma atleta que não apresentava inicialmente alteração evoluiu com o padrão de discinesia, resultando na prevalência acumulada de 10 atletas (83\%) nesta população. Conclusão: Concluiu-se que uma única sessão de treinamento foi capaz de aumentar a prevalência de discinesia escapular em 8\% (ou uma atleta) das atletas de voleibol.

Palavras-chave: voleibol, discinesias, escápula, fadiga.

\section{ABSTRACT}

Introduction: In the throwing motion, the balance of scapulothoracic rhythm plays a key role in the efficiency of the Sporting gesture. Scapular diskinesis is commonly associated to specific injuries of the throwing shoulder. Among the causes of scapular dyskinesia, muscle fatigue is often reported due to overuse of the periescapular muscles. Objective: To evaluate the influence of training in the presence of scapular dyskinesis in volleyball athletes. Methods: This is a prospective cohort study in which twelve female athletes practicing volleyball, who had no previous surgeries on the shoulders and practicing at least 10 hours of weekly workouts were evaluated. Assessment pre and post workout was performed, which consisted of $30 \mathrm{~min}$ warm up and $120 \mathrm{~min}$ of specific training. Scapular dyskinesia was evaluated as 'YES or 'NO' as for the presence or absence of abnormalities in the scapular-thoracic movement, respectively. Results: As a result of this study we identified a prevalence of scapular dyskinesis in nine athletes from twelve in the pre-training (75\%) and after the training session only one athlete evolved with standard dyskinesia, resulting in the cumulative prevalence of 10 athletes (83\%) in this population. Conclusion: This study concluded that a single training session was able to increase the prevalence of scapular dyskinesia in 8\% (or one athlete) of volleyball players.

Keywords: volleyball, dyskinesias, scapula, fatigue.

\section{RESUMEN}

Introducción: En el movimiento de ataque, el equilibrio del ritmo escapulotorácico ejerce un papel fundamental en la eficiencia del gesto deportivo, siendo la disquinesia escapular comúnmente asociada a lesiones específicas del hombro del lanzador. Entre sus causas es frecuentemente relatada la fatiga muscular como consecuencia por el uso excesivo de la musculatura periescapular. Objetivos: Evaluar la influencia del entrenamiento en la presencia de la disquinesia escapular en atletas de voleibol. Métodos: Se trata de un estudio de cohorte prospectivo, en el que fueron evaluadas 12 atletas del género femenino practicantes de voleibol, que no poseían cirugías previas en los hombros y practicaban como mínimo 10 horas de entrenamientos semanales. Fueron realizadas evaluaciones, antes y después del entrenamiento, que tenían 30 minutos de calentamiento y 120 minutos de entrenamiento específico de la modalidad. La disquinesia escapular fue graduada en "SI" O "NO", definida de acuerdo con la presencia o ausencia de alteraciones en el estándar de movimiento escapulotorácico, respectivamente. Resultados: La prevalencia 
en esta población fue identificada en nueve atletas en la evaluación antes del entrenamiento (75\%) y después de la realización del entrenamiento una atleta que no presentaba alteración inicialmente evolucionó con el estándar de disquinesia, resultando en la prevalencia acumulada de 10 atletas (83\%) en esta población. Conclusión: Se concluye que una única sesión de entrenamiento fue capaz de aumentar la prevalencia de disquinesia escapular en 8\% (0 una atleta) de las atletas de voleibol.

Palabras clave: voleibol, disquinesias, escápula, fatiga.

\section{INTRODUÇÃO}

Entre os gestos desempenhados com o membro superior na pratica do voleibol, o movimento de ataque com o braço dominante acima da cabeça repercute em diversas lesões no complexo do ombro, gerando em média 6,5 semanas de afastamento de treinamentos ou competições ${ }^{1}$. No esporte, sua incidência tem sido estimada entre 8\% a $20 \%$, sendo a terceira condição mais comum em atletas de voleibol e a segunda maior relacionada ao overuse $e^{2,3}$.

No movimento de ataque, o equilíbrio do ritmo escápulotorácico exerce função fundamental na eficiência do gesto esportivo promovido principalmente pela sinergia no padrão de ativação muscular do manguito rotador e músculos periescapulares, bem como proporcionando uma base estável e facilitando a transmissão de forças pela cadeia cinética. Alterações no movimento e do posicionamento escapulotorácico estão comumente associadas a lesões específicas do ombro do arremessador, tais como impacto interno, instabilidade, lesões labrais e do manguito rotador, levando assim a condição intitulada de discinesia escapular ${ }^{4,5}$. Esta condição foi relatada em $100 \%$ dos pacientes com instabilidade glenoumeral, 94\% dos pacientes com lesão do lábio glenoidal e $68 \%$ dos pacientes com lesão do manguito rotador ${ }^{6}$.

Dentre as causas da discinesia escapular, a fadiga muscular consequente ao uso excessivo da musculatura periescapular é comumente identificada no ombro dominante de arremessadores, entretanto pesquisas recentes demonstraram alterações no movimento e posicionamento em associações com diminuição na flexibilidade, alterações posturais, desequilíbrios musculares e alterações neurológicas, dentre outras já citadas acima-10.

Para avaliação destas alterações, diversas pesquisas desenvolveram métodos de mensuração a fim de melhor entende-las. Análises bidimensionais e posturas estáticas foram as primeiras estudadas, entretanto os métodos tridimensionais mostraram-se confiáveis, contudo, inviáveis na prática clínica pelo alto custo e por utilizar técnicas invasivas para mensuração. Recentemente, pesquisadores utilizam a avaliação clínica somente pela observação do movimento escapular que, mostrou-se sensível (78\%) para identificação da presença ou não de discinesia escapular, porém pouco específica (38\%) para suas causas, estabelecendo assim a denominação do método do "SIM" ou "NÃO"5,6,11,12. Baseado na importância da escápula no gesto esportivo de atletas que utilizam movimentos do membro superior acima da cabeça e da escassez da literatura sobre a relação da discinesia e o treinamento específico nesta modalidade.

O objetivo do presente estudo foi avaliar a influência de uma única sessão de treinamento na prevalência de discinesia escapular em atletas de voleibol.

\section{MATERIAIS E MÉTODOS}

Estudo do tipo observacional do tipo coorte prospectivo baseado no Stregthening the Reporting of Observational Studies in Epidemiology STROBE $E^{13}$ que incluiu atletas jovens praticantes de voleibol. O estudo foi aprovado pelo Comitê de Ética em Pesquisa da Universidade Federal de São Paulo, SP, Brasil, com o número de registro 283.280.
Foram convidados a participar do estudo atletas jovens de 17 a 19 anos, do gênero feminino praticantes de voleibol com pelo menos quatro anos de treinamento competitivo em um centro de cidade de TaubatéSP, Brasil. Preencheram os critérios de inclusão atletas que estavam em treinamento regular sem restrições de volume, carga de treinamento ou em tratamento fisioterápico, não possuíam cirurgias prévias nos ombros e que praticavam no mínimo 10 horas de treinos semanais. Foram excluídos do estudo os atletas que não conseguiram terminar o treino proposto por dor ou lesão. As atletas que possuíam mais de 18 anos e concordaram em participar do estudo assinaram um Termo de Consentimento Livre e Esclarecido (TCLE). As participantes que possuíam menos de 18 anos assinaram o Termo de Assentimento e seus responsáveis o TCLE.

O estudo teve duração de um dia, o qual as atletas foram recrutadas para avaliação 30 minutos prévios ao início do treino e, em seguida realizaram as atividades propostas pelo preparador físico e técnico, no qual continham 30 minutos de aquecimento e 120 minutos de treino específico da modalidade. Imediatamente após o término as participantes foram reavaliadas, sendo que para que não houvesse diferença entre a primeira e a última a ser reavaliada foi mantido o treino específico de ataque e defesa durante a espera.

As atletas selecionadas a partir dos critérios de inclusão passaram por uma única avaliação, que consistiu primeiramente em uma anamnese para coleta de dados demográficos e histórico de lesões prévias. Em seguida foi realizada a avaliação da presença de discinesia escapular.

\section{Avaliação inicial}

Os participantes responderam ao fisioterapeuta responsável pela equipe questões para obtenção de dados de idade, altura, peso, histórico da prática do esporte, tempo de prática, dominância, posição, histórico de lesões musculoesqueléticas e dor atual. No histórico de lesões, foi questionado se a participante já teve alguma lesão nos ombros relacionada a sua prática esportiva, o local de lesão e se teve que passar por algum procedimento cirúrgico nos ombros.

Todos os sujeitos receberam informações sobre a realização do teste que consistia na elevação bilateral dos braços sincronicamente no plano escapular. A atleta foi posicionada de costas para o avaliador e ao lado da mesma foram colocados duas hastes de $210 \mathrm{~cm}$ de altura em um ângulo de $60^{\circ}$ que correspondia ao plano escapular. Para padronização foram demarcados a distância do sujeito à parede $(110 \mathrm{~cm})$, câmera ao atleta $(115 \mathrm{~cm})$, altura da câmera $(170 \mathrm{~cm})$ e a distância entre as hastes $(65 \mathrm{~cm})$. Previamente o avaliador demonstrou a maneira correta de realizar os movimentos e foi solicitado que cada atleta realizasse e, somente quando estavam sendo realizados com a velocidade e angulação adequadas foi iniciada a filmagem. Foram solicitadas 10 repetições do movimento, porém os cinco primeiros foram descartados por serem considerados como aprendizado 6 (figura 1.)

A discinesia escapular foi definida como a presença de alterações no padrão de movimento escapulotorácico como disritmia, compensações e desequilíbrios musculares visualmente percebidos ${ }^{14}$. A discinesia 


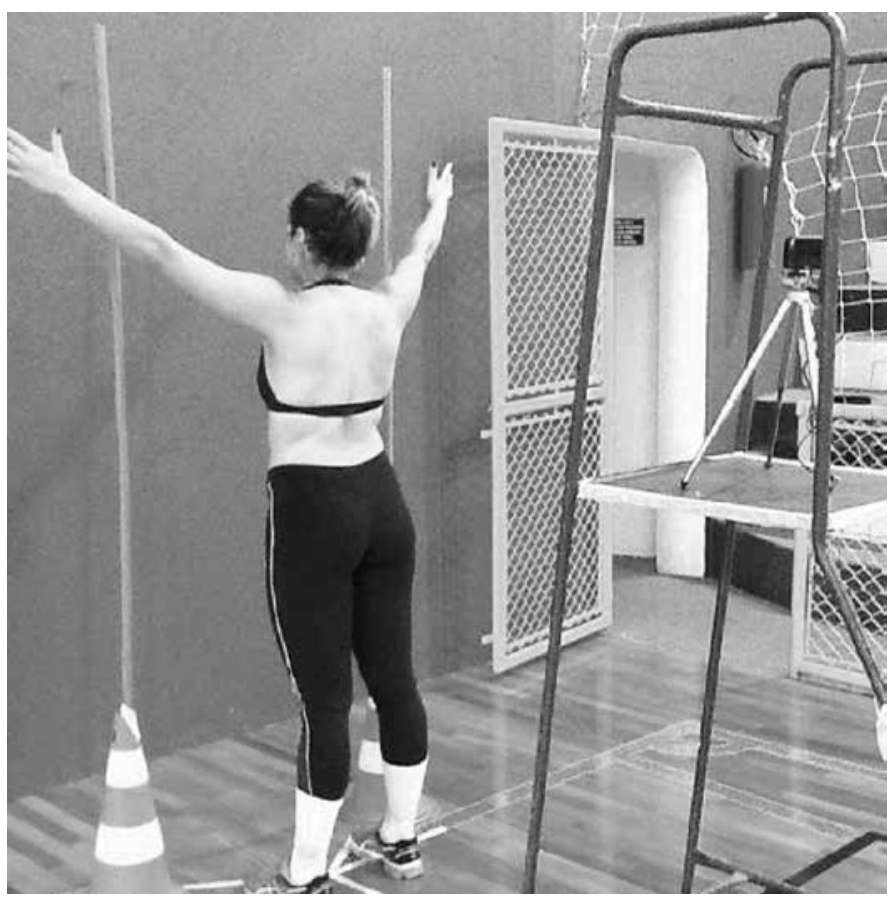

Figura 1. Avaliação da discinesia escapular pelo método do SIM ou NÃO.

foi graduada em "SIM ou NÃO", similar ao método utilizado por Kibler et al. ${ }^{5}$ em 2009. Este método mostrou-se com uma boa validade e confiabilidade intra-observadores e também quando comparados à análise em vídeo 3D ${ }^{11}$. A presença ou não de discinesia foi identificada para cada atleta, independente do lado, caso identificado de forma bilateral somente uma alteração foi registrada.

A sequência de teste foi gravada por uma câmera de vídeo (Sony, cyber-shot DSC-w35) e dois avaliadores independentes registraram seus resultados e em consenso definiram a presença ou não de discinesia pré e pós-treino nesta população.

\section{Análise de dados}

Primeiramente realizou-se uma análise descritiva com os dados demográficos (idade, altura, peso, dominância de membros superiores (MMSS), categoria do esporte, a frequência de treino, tempo de prática e histórico de lesões) em média e desvio padrão. Para análise da variável de interesse foi realizado o cálculo do percentual da presença de discinesia escapular das atletas antes e após o treinamento realizado.

\section{RESULTADOS}

Foram incluídas no estudo 12 atletas com média de idade de 18,3 $( \pm 0,6)$ anos, altura de $1,78( \pm 0,5) \mathrm{m}$, massa corporal de $74,5( \pm 10,2) \mathrm{Kg}$, IMC $23,6( \pm 2,4) \mathrm{kg} / \mathrm{m}^{2}$, e tempo de prática de $6,8( \pm 1,8)$ anos. Todos os atletas possuíam o lado direito como dominante para a prática esportiva. A descrição completa dos dados demográficos em relação ao grupo estudado pode ser melhor observada na tabela 1.

Tabela 1. Descrição dos participantes do estudo.

\begin{tabular}{c|c}
\hline Variáveis & $\mathbf{n}=\mathbf{1 2}$ \\
\hline Idade $(\mathrm{a})$ & $18,3( \pm 0,6)$ \\
\hline Estatura $(\mathrm{m})$ & $1,78( \pm 0,5)$ \\
\hline Massa corporal $(\mathrm{Kg})$ & $74,5( \pm 10,2)$ \\
\hline $\mathrm{IMC}\left(\mathrm{Kg} / \mathrm{m}^{2}\right)$ & $23,6( \pm 2,4)$ \\
\hline Dominância membro superior & $12(100 \%)$ \\
\hline Direita & $6,8( \pm 1,8)$ \\
\hline Experiência no esporte (anos) & $6( \pm 0)$ \\
\hline Treinos semanais (dias) & $180( \pm 0)$ \\
\hline Minutos por treino &
\end{tabular}

A prevalência de discinesia escapular foi identificada em nove atletas na avaliação pré-treino (75\%) e após a realização do treinamento, uma atleta que não apresentava alteração inicialmente evoluiu com o padrão de discinesia, resultando na prevalência acumulada de 10 atletas (83\%) nesta população.

\section{DISCUSSÃO}

Atualmente, diversos métodos são utilizados para avaliação escapular, entre os quais, a avaliação tridimensional, que consiste no uso de um sistema eletromagnético para análise cinemática do movimento, 0 padrão ouro para identificação das alterações na posição e movimento escapulotorácico, por conferir maior especificidade á avaliação, dada a prevalente natureza multidimensional da discinesia escapular ${ }^{12,15-17}$. A avaliação SIM/NÃO, utilizada no presente estudo, baseia-se, entretanto, na observação do padrão dinâmico da escápula em um único plano, método este que impõe alguma dificuldade em detectar padrões específicos da discinesia escapular, devido á freqüente assimetria em múltiplos planos. Embora possua baixa especificidade, mostrou um nível médio de precisão e alto nível de sensibilidade e concordância em um estudo realizado por Kibler et al. ${ }^{5}$ de correlação de confiabilidade entre métodos observacionais de avaliação clínica da discinesia escapular e o método de avaliação tridimensional realizado. O mesmo estudo mostrou ainda que o método sim/não diminui o risco de achados falso negativo, por indicar sujeitos que realmente possuem discinesia. Tal combinação indica que este método é uma boa ferramenta examinadora e prove boa confiabilidade entre observadores em suas avaliações observacionais da discinesia da escápula ${ }^{5}$.

Este estudo mostrou um aumento de 8\% de prevalência de discinesia escapular entre a avaliação pré-treino, que identificou a condição prévia em $75 \%$ das atletas avaliadas e a avaliação pós treino, que apontou uma prevalência acumulada de $83 \%$ da população, a qual foi composta por 12 atletas do sexo feminino com média de idade de 18,3 e tempo de prática de 6,8 anos. Todas as atletas possuíam o lado direito como dominante para a prática esportiva e o treino teve a duração de 180 minutos. Um estudo semelhante, realizado com nadadores, apontou aumento progressivo na prevalência de discinesia escapula quanto maior o volume de treino em períodos consecutivos, visto que o treino foi segmentado em quatro fases, com aumento gradual na intensidade do trabalho aeróbico, entre as quais foram realizadas quatro avaliações. A prevalência de discinesia escapular em atletas isentos de sintomas prévios relacionados, foi de $37 \%, 68 \%, 73 \%$ e $82 \%$ respectivamente nas quatro avaliações realizadas. Tais achados reafirmam o resultado obtido pelo presente estudo, no entanto, há de se ressaltar algumas diferenças relevantes, entre as quais, a menor proporção entre a prevalência acumulada, o que pode ser atribuído ao critério de inexistência prévia de sintomas entre os avaliados, a intensidade da sobrecarga imposta, a extensão do treino e o número de participantes recrutados ${ }^{6}$.

No estudo de Joshi et al. ${ }^{18}$ foi utilizado um protocolo específico de fadiga que envolveu exercícios de rotação externa com o membro superior dominante de atletas que utilizam movimentos de membro superior acima da cabeça, realizada entre $0^{\circ}$ a $75^{\circ} \mathrm{com}$ halteres, estando o membro superior posicionado a $90^{\circ}$ e o tronco estabilizado. Os participantes realizaram repetições destes exercícios até que o ponto em que o ritmo do movimento não pode ser mantido, com intervalos de 30 segundos entre as séries. As séries foram repetidas até que o número fosse inferior a 50\% das repetições realizadas na primeira série. Os participantes foram considerados fadigados quando a média do pico de força da rotação externa diminuísse a menos de 25\% da linha de base do pico de força. $O$ estudo concluiu que a fadiga dos rotadores externos causou alteração na ativação e cinemática dos músculos 
escapulares. Já no estudo realizado por Tripp et al. ${ }^{19}$, jogadores de baseball eram reavaliados após realizarem 20 arremessos com velocidade máxima e apenas realizavam a avaliação pós intervenção quando referiam nível 14 da escala de Borg. O estudo concluiu que a fadiga funcional leva a perda da acuidade dos movimentos do membro superior e promove efeitos deletérios que podem levar a implicações patologias na articulação. No estudo de Madsen et al. ${ }^{6}$ um treino progressivo de natação com a duração de 100 minutos, segmentado em 4 intervalos, com aumento de carga gradual em relação ao trabalho aeróbico foi suficiente para mostrar que a prevalência de discinesia escapular após um treino normal de natação é alta e que progride quanto maior a carga de treino a que é submetido o atleta. A diferença quanto á prevalência de discinesia entre a primeira e última avaliação foi de $45 \%$. Em nosso estudo somente o treino geral de vôlei, sem exigências de volume e intensidade pode-se observar um aumento da prevalência da discinesia escapular, possivelmente devido ao uso unilateral do membro superior no esporte estudado o que gera, contudo, sobrecarga na musculatura pelo gesto repetitivo. No entanto a diferença entre a prevalência de discinesia escapula encontrado entre as avaliações pré e pós treino foi de 8\%. A diferença entre a significância estatística encontrada entre os estudos se deve, provavelmente, á intensidade e especificidade dos treinos aplicados.

Os resultados obtidos por este estudo fundamentam a necessidade de se investir em identificação e intervenção precoce na discinesia escapular, em trabalhos preventivos e de reabilitação do ombro, bem como em trabalhos de resistência em ombros de atletas de voleibol ${ }^{19,20}$.

A maior limitação do estudo foi a restrição quantitativa da população avaliada. Outros importantes fatores devem ser considerados para maior precisão e significância nos resultados em estudos futuros, quais sejam, uma maior especificidade no treino, a exemplo um treino intensivo de ataque, bem como uma maior intensidade de sobrecarga de trabalho específico para a estrutura avaliada e uma maior extensão do treino, a fim de promover maior fadiga dos músculos periescapulares para avaliar sua influência na discinesia escapular. Critérios de inclusão mais restritivos, tais como a inexistência prévia de dor e lesão no ombro podem também ser relevantes. Finalmente, sugere-se avaliações após um trabalho específico de sobrecarga para os músculos estabilizadores da escápula, com um número de participantes mais significativo, envolvendo outras populações de atletas que fazem uso repetido de movimentos dos membros superiores acima da cabeça.

\section{CONCLUSÃO}

Uma única sessão de treinamento foi capaz de aumentar a prevalência de discinesia escapular em 8\% (ou uma atleta) das atletas de voleibol.

Todos os autores declararam não haver qualquer potencial conflito de interesses referente a este artigo.

\section{REFERÊNCIAS}

1. Reeser JC, Verhagen E, Briner WW, Askeland TI, Bahr R. Strategies for the prevention of volleyball related injuries. Br J Sports Med. 2006;40(7):594-600.

2. Reeser JC, Joy EA, Porucznik CA, Berg RL, Colliver EB, Willick SE. Risk factors for volleyball-related shoulder pain and dysfunction. PM R. 2010;2(1):27-36.

3. Verhagen EA, Van der Beek AJ, Bouter LM, Bahr RM, Van Mechelen W. A one season prospective cohort study of volleyball injuries. Br J Sports Med. 2004;38(4):477-81.

4. Kibler WB, Sciascia A. Current concepts: scapular dyskinesis. Br J Sports Med. 2010;44(5):300-5

5. Kibler WB, Ludewig PM, McClure P, Uhl TL, Sciascia A. Scapular Summit 2009: introduction. July 16, 2009, Lexington, Kentucky. J Orthop Sports Phys Ther. 2009;39(11):A1-A13.

6. Madsen PH, Bak K, Jensen S, Welter U. Training induces scapular dyskinesis in pain-free competitive swimmers: a reliability and observational study. Clin J Sport Med. 2011;21(2):109-13.

7. Borstad JD, Ludewig PM. Comparison of scapular kinematics between elevation and lowering of the arm in the scapular plane. Clin Biomech (Bristol, Avon). 2002;17(9-10):650-9.

8. Burkhart SS, Morgan CD, Kibler WB. The disabled throwing shoulder: spectrum of pathology Part I: pathoanatomy and biomechanics. Arthroscopy. 2003;19(4):404-20

9. Laudner KG, Myers JB, Pasquale MR, Bradley JP, Lephart SM. Scapular dysfunction in throwers with pathologic internal impingement. J Orthop Sports Phys Ther. 2006;36(7):485-94.

10.Timmons MK, Thigpen CA, Seitz AL, Karduna AR, Arnold BL, Michener LA. Scapular kinematics and subacromial-impingement syndrome: a meta-analysis. J Sport Rehabil. 2012;21(4):354-70
11. McClure P, Tate AR, Kareha S, Irwin D, Zlupko E. A clinical method for identifying scapular dyskinesis, part 1: reliability. J Athl Train. 2009;44(2):160-4.

12. McClure PW, Michener LA, Sennett BJ, Karduna AR. Direct 3-dimensional measurement of scapular kinematics during dynamic movements in vivo. J Shoulder Elbow Surg. 2001;10(3):269-77.

13. Von Elm E, Altman DG, Egger M, Pocock SJ, Gøtzsche PC, Vandenbroucke JP, et al. The Strengthening the Reporting of Observational Studies in Epidemiology (STROBE) statement: guidelines for reporting observational studies. Prev Med. 2007;45(4):247-51

14. Borloz S, Graf V, Gard S, Ziltener JL. Scapular dyskinesis. Rev Med Suisse. 2012;8(367):2422-8.

15. Ludewig PM, Cook TM. Alterations in shoulder kinematics and associated muscle activity in people with symptoms of shoulder impingement. Phys Ther. 2000;80(3):276-91.

16. van Andel C, van Hutten K, Eversdijk M, Veeger D, Harlaar J. Recording scapular motion using an acromion marker cluster. Gait Posture. 2009;29(1):123-8.

17. Yano Y, Hamada J, Tamai K, Yoshizaki K, Sahara R, Fujiwara T, et al. Different scapular kinematics in healthy subjects during arm elevation and lowering: glenohumeral and scapulothoracic patterns. J Shoulder Elbow Surg. 2010;19(2):209-15.

18. Joshi M, Thigpen CA, Bunn K, Karas SG, Padua DA. Shoulder external rotation fatigue and scapular muscle activation and kinematics in overhead athletes. J Athl Train. 2011;46(4):349-57.

19. Tripp BL, Yochem EM, UhI TL. Functional fatigue and upper extremity sensorimotor system acuity in baseball athletes. J Athl Train. 2007:42(1):90-8

20. Kugler A, Krüger-Franke M, Reininger S, Trouillier HH, Rosemeyer B. Muscular imbalance and shoulder pain in volleyball attackers. Br J Sports Med. 1996;30(3):256-9. 